\title{
Therapeutic Effects of Umbilical Cord Blood Derived Mesenchymal Stem Cell-Conditioned Medium on Pulmonary Arterial Hypertension in Rats
}

\begin{abstract}
Jae Chul Lee ${ }^{1,2,3} \cdot$ Choong Ik Cha ${ }^{3}$ Dong-Sik $\mathrm{Kim}^{2}$. Soo Young Choe ${ }^{1}$

'Department of Biology, School of Life Sciences, Chungbuk National University, Cheongju, ${ }^{2} \mathrm{De}$ partment of Surgery, Brain Korea 21 PLUS Project for Medical Sciences and HBP Surgery and Liver Transplantation, Korea University College of Medicine, Seoul; ${ }^{3}$ Department of Anatomy, Seoul National University College of Medicine, Seoul, Korea
\end{abstract}

Received: September 4, 2015 Accepted: September 9, 2015

\section{Corresponding Authors}

Soo Young Choe, PhD

Department of Biology, School of Life Sciences,

Chungbuk National University, 1 Chungdae-ro,

Seowon-gu, Cheongju 28644, Korea

Tel: +82-43-261-2297

Fax: +82-43-275-2291

E-mail: leejc@chungbuk.ac.kr

Dong-Sik Kim, MD, PhD

Department of Surgery, Brain Korea 21 PLUS Project for Medical Sciences and HBP Surgery and Liver Transplantation, Korea University College of Medicine, 145 Anam-ro, Seongbuk-gu, Seoul 02841, Korea

Tel: +82-2-2286-1431

Fax: +82-2-2286-1428

E-mail: beas100@korea.ac.kr
Background: Human umbilical cord blood-derived mesenchymal stem cells (hUCB-MSCs) may have multiple therapeutic applications for cell based therapy including the treatment of pulmonary artery hypertension $(\mathrm{PAH})$. As low survival rates and potential tumorigenicity of implanted cells could undermine the mesenchymal stem cell (MSC) cell-bas ropy, we chose to investigate the use of conditioned medium (CM) from a culture of $N$ feasible alternative. Methods: CM was prepared by culturing hUCB-MSCs in t. model of PAH induced by monocrotaline, we infused media via the tail-vein of 6-week-old Sprague-Daw unconditioned media, CM infusion reduced th ventricle+interventricular septum) ratio, and mals. Also, the number of interleukin $1 \alpha$ (l

\section{raine} chemokin sue inhibitor of metalloproteinase 1 (TK number of terminal deoxynucleotid ansferase rated deoxyuridine triphosphate nick-end labeling technique (TUNEL)-posit cells decreasea, nificantly in the CM treated animals. Conclusions: From our in vivo data e rat model, the observed decreases in the TUNEL staining suggest a potential therapeutic $b$ of the in ameliorating PAH-mediated lung tissue damage. Increased IL-1 $1 \alpha$, and TIM may play important roles in this regard. ira function in the treated aninotif) ligand 5 (CCL5), and tisspheroids. In a rat conditioned culture ared with the control pressu, the right ventricle/(left

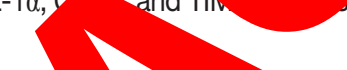

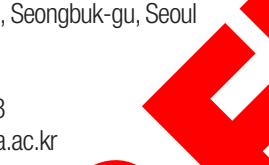

ey Woru. Apoptosis; Culture media, conditioned; Gene expression; Mesenchymal stromal cells; Pumonary artery hypertension

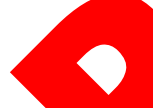

Pulmonary artery hyp von ( $\mathrm{PAH})$ is a progressive chronic disease with a high mortali, ate. ${ }^{1} \mathrm{PAH}$ has a complex disease mechanism, but its cardinal signs are an elevation of pulmonary artery pressure, right ventricular (RV) hypertrophy, and arteriolar wall remodeling. ${ }^{2}$ Increased pulmonary vascular resistance and over-proliferation of pulmonary artery endothelial cells leads to remodeling of the pulmonary vasculature. ${ }^{3-5}$ There is also damage to the pulmonary microvasculature impacting the blood flow from the heart to the lungs. ${ }^{6,7}$ Although current treatments may prolong and improve quality of life for the patients, the long-term prognosis for $\mathrm{PAH}$ is poor with a 2- to 3-year survival at the time of diagnosis. ${ }^{1}$
Autologous implantation of bone marrow mononuclear cells, known to be enriched in mesenchymal stem cells (MSCs), has demonstrated safety and effectiveness in therapeutic angiogenesis. ${ }^{8}$ A number of studies have also indicated a therapeutic benefit from bone marrow derived MSCs in increasing respiratory function in animal models of PAH. ${ }^{9,10}$ In separate studies, human umbilical cord blood-derived MSCs (hUCB-MSCs) have also improved lung function in animal models of $\mathrm{PAH}$ and in a number of human PAH patients. ${ }^{11-13}$

In previous studies, we demonstrated the neuroprotective potential of various conditioned media (CM), namely human adipose tissue-derived stem cell (hADSC)-conditioned media and 
human neural stem cell (hNSC)-conditioned media to treat rats with stroke and Huntington's disease. ${ }^{14,15}$ We also investigated gene expression changes by microarray analysis after injection of hUCB-MSCs into rats in an experimental model of PAH. ${ }^{16}$ Based on our findings from that study, we undertook an investigation to assess the feasibility and safety of conditioned medium from hUCB-MSCs (hUCB-MSC-CM) in the same rat PAH model. We also tested the hypothesis that the conditioned media from these cells may lead to improved lung function in the affected rats. Here, we elaborate on our results and demonstrate that the conditioned media provides a therapeutic benefit in the rat model of PAH. As there are certain advantages in using conditioned media in lieu of autologous whole bone marrow or umbilical cord cells as sources for MSCs, our data may be provide a means of increasing the accessibility of MSCs to treat various diseases including PAH.

\section{MATERIALS AND METHODS}

\section{Animals}

Six-week-old male Sprague-Dawley rats were used. All rat were housed in climate-controlled conditions with a 12-he light/12-hour dark cycle, and had free access to food and wate. All animal experiments were approved by the appron tutional Review Boards of the Seoul National $y$ ersi College of Medicine (Seoul, Korea; SNU-101122 2) a the Care Use of Laboratory Animals (NI as is No. $8,-23$, revised in 1996).

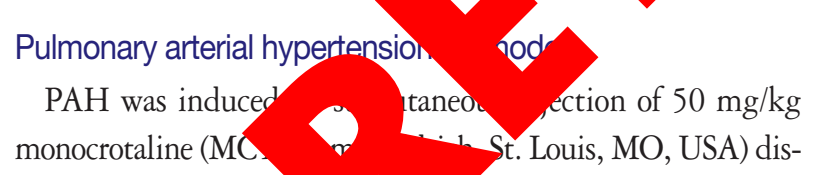
solved in $0.5 \mathrm{~N} \mathrm{HCl}$. Th were grouped into a control group (C group) $(\mathrm{n}=20)$, injectio $\alpha \alpha$-minimal essential medium ( $\alpha$ MEM) followed by MCT group (M group) $(n=20)$, and injection of MCT followed by hUCB-MSC-CM transfusion group (CM group) ( $\mathrm{n}=20)$. $\alpha \mathrm{MEM}$ and hUCB-MSC-CM $(0.5 \mu \mathrm{L} / \mathrm{hr})$ were transfused by tail-vein 7 days after MCT injection. The animals were sacrificed at 7, 14, 21, and 28 days after hUCBMSC-CM transfusion. Tissues were removed and immediately frozen at $-70^{\circ} \mathrm{C}$ for enzyme analysis.

\section{Cell preparation and culture of hUCB-MSCs}

hUCB-MSCs were obtained from the Biomedical Research Institute (Seoul, Korea). Isolated human MSCs were expanded in culture as previously described. ${ }^{6}$ hUCB-MSCs were maintained in aMEM (Gibco, Grand Island, NY, USA) supplemented with $10 \%$ fetal bovine serum (Gibco), $100 \mathrm{U} / \mathrm{mL}$ penicillin (Gibco), and $100 \mathrm{~g} / \mathrm{mL}$ streptomycin (Gibco). Passages up to 5 were used for experiments.

\section{Preparation of hUCB-MSC-CM}

To generate hUCB-MSC-CM spheroids, ${ }^{16,17} 30 \mu \mathrm{L}$ of cell suspension $\left(1 \times 10^{6}\right.$ cells $\left./ \mathrm{mL}\right)$ were applied to the lid of a Petri dish containing phosphate buffered saline (PBS). After 24 hours of incubation, spheroids formed in were retrieved. For the three-dimensional bioreac altur CB-MSC spheroids $\left(4.2 \times 10^{7}\right.$ cells) were cultyred $\mathrm{T}$. co, Vineland, NJ, US Antainin at $70 \mathrm{rpm}$. To obtain medius, was changed to $\alpha \mathrm{MEM}$ without seruy the altured for 2 days. CM was collected gation.

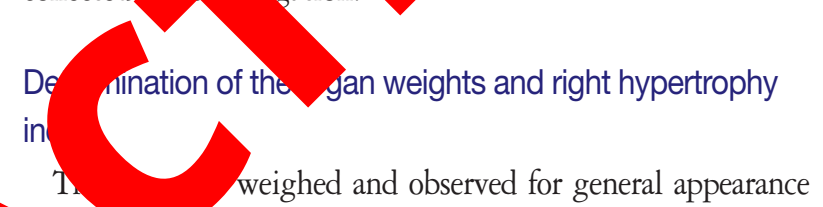
ring the study period. The animals were sacrificed at the time. The wet weights of the excised right ventricle $(\mathrm{RV})$, left ventricle (LV), and interventricular septum (IVS) were heasured. The weights of the LV and IVS were added (LV + IVS) to determine the RV to $\mathrm{LV}+\mathrm{IVS}$ ratio [RV/(LV + IVS)], which was used to determine the right hypertrophy index.

\section{Pulmonary hemodynamics}

Rats were anaesthetized by intraperitoneal injection of urethane and secured on a surgical stage. An 8-mm-long right internal jugular vein was isolated and ligated at the distal end. The vessel was cut at the proximal end of ligation. A catheter filled with heparinized saline was rapidly inserted along the incision and slowly advanced for about $5 \mathrm{~cm}$ to enter the pulmonary artery. The standard of pulmonary hypertension was defined as a systolic pulmonary pressure (SPAP) larger than $50 \mathrm{~mm} \mathrm{Hg} .^{18}$ Hemodynamic parameters were recorded at baseline and at 7 , 14,21 , and 28 days.

\section{Immunohistochemistry}

Excised lung tissues were incubated overnight in $10 \%$ buffered formalin. Four-micrometer-thick sections were cut from paraffin embedded tissue blocks, deparaffinized in xylene, and rehydrated in graded alcohol solutions $(70 \%-100 \%)$. Heat antigen retrieval was achieved by boiling the tissue sections in an- 
tigen retrieval solution in $\mathrm{pH} 6.0$ or $\mathrm{pH} 9.0$ (Dako, Carpinteria, CA, USA) for 10 minutes in a microwave prior to incubation at $4^{\circ} \mathrm{C}$ overnight with primary antibodies against interleukin $1 \alpha$ (IL-1 $\alpha$ ), chemokine (C-C motif) ligand 5 (CCL5), and tissue inhibitor of metalloproteinase 1 (TIMP-1; Abcam, Cambridge, MA, USA). After incubation with the appropriate biotinylated secondary antibodies for 30 minutes at $4{ }^{\circ} \mathrm{C}$ and subsequently with streptavidin (Dako, Kyoto, Japan), color development was done using diaminobenzidine (DAB) as a chromogen and counterstained with hematoxylin.

\section{Western blot analysis}

The tissue was homogenized in $10 \mathrm{mM}$ Tris $\mathrm{HCl}$ buffer, $\mathrm{pH}$ 7.4 containing $0.5 \mathrm{mM}$ ethylenediaminetetraacetic acid, $\mathrm{pH}$ 8.0, $0.25 \mathrm{M}$ sucrose, $1 \mathrm{mM}$ phenylmethylsulfonyl fluoride, 1 $\mathrm{mM} \mathrm{Na} \mathrm{VO}_{3}$, and a protease inhibitor cocktail (Roche-Boehringer-Mannheim, Mannheim, Germany). After centrifugation, the supernatant was subjected to sodium dodecyl sulfate polyacrylamide gel electrophoresis (SDS-PAGE). Samples equivalent to $25 \mu \mathrm{g}$ of protein content were loaded and size-separated by $8 \%-12 \%$ SDS-PAGE. The proteins on the acrylamide gel were transferred to a polyvinylidene difluoride membrane ( $\mathrm{M}$ lipore, Bedford, MA, USA) at $400 \mathrm{~mA}$ in a transfer buffer con taining $25 \mathrm{mM}$ Tris and $192 \mathrm{mM}$ glycine, $\mathrm{pH} 8$. cellulose membrane was blocked in tris-buffered non-fat dry milk at room temperature for $1 \mathrm{~b}$ 20 and incubated with the appropriated ary antio including anti-IL-1 $\alpha$ (Santa Cruz Biote on vista Crus, CA, USA), anti-CCL5 (Fitzgerald Ind res Interna Concord, MA, USA), anti-TIMP-1 (A anti-caspase-3, anti$\mathrm{Bcl}-2$, anti-actin (Santa Canz Bro olo $>4^{\circ} \mathrm{C}$ for overnight. The membrar hen in with horseradish peroxidase-conjuga nology, Danvers, MA, D 1 hour at room temperature. After washing, the membran nescent ECL-detection kit from GE-Healthcare (Piscataway, NJ, USA).

\section{Cytokine array and gene expression in lung tissues}

The lung samples were collected at termination (4 days after hUCB-MSC-CM injection) and quickly frozen in liquid nitrogen. A rat cytokine array (ARY008, R\&D Systems, Minneapolis, MN, USA) was used to screen the lung homogenates according to the manufacturer's instructions. The samples were pooled per treatment group and equal amounts of protein were loaded on the blots.
In situ terminal deoxynucleotidyl transferase-mediated deoxyuridine triphosphate nick-end labeling technique assay for lung cell apoptosis

Apoptotic cells in the tissue sections were detected by the terminal deoxynucleotidyl transferase-mediated deoxyuridine triphosphate nick-end labeling technique (TUNEL) using a commercial apoptosis kit (TACS TM TdT Kit, R\&D Systems), according to the supplier's instructions. In brief, the lung tissue sections were de-paraffinized with xylene and ethanol and rinsed with PBS. The sections were then treated with proteinase $\mathrm{K}$ in PBS followed by quenching of en peroxidase. A biotinylated dNTP mix was adde the ends of DNA by terminal deoxynucleotidy tra se (T) After incubating with streptavidin-ho dish pu the sections were stained with DAR an stained wh methyl green. Finally, the section dehanol, cleared with xylene, and moup coverslips a permanent medium. According to supplic ructions, experimental controls included for assay were TA nuclease-treated thyroid tissue sections as positive ontrol and the omission of the $\mathrm{TdT}$ reaction step ive control.

Results were expressed as the mean \pm standard deviation. An anpaired two-tailed $t$ test and Mann-Whitney test were used, and a p-value less than .05 was considered statistically significant. SPSS ver. 14.0 for Windows (SPSS Inc., Chicago, IL, USA) was used for all statistical analyses.

\section{RESULTS}

Changes in body and organ weights and systolic pulmonary artery pressure after injection with hUCB-MSC-CM in $\mathrm{PAH}$ rats

hUCB-MSC-CM has the potential to increase cell differentiation and induce immune modulation in various disease models. ${ }^{19,20}$ However, the role of hUCB-MSC-CM in PAH has not been well elucidated. To address this, in our rat model of $\mathrm{PAH}$, following MCT treatment, we treated rats with hUCB-MSC$\mathrm{CM}$ and sham treated for the control group. There was a significant decrease in body weight at 14,21, and 28 days in the MCT group ( $\mathrm{M}$ group) compared to the control group ( $\mathrm{C}$ group). However, body weight increased at 21 and 28 days in the conditioned media treated group (CM group) compared to the $\mathrm{M}$ group. The $\mathrm{M}$ group also showed increased weights of the RV at 21 and 28 days. The sum weight of LV + IVS was not signifi- 
Table 1. Changes of body and organ weights after hUCB-MSCS-CM injection in PAH rats

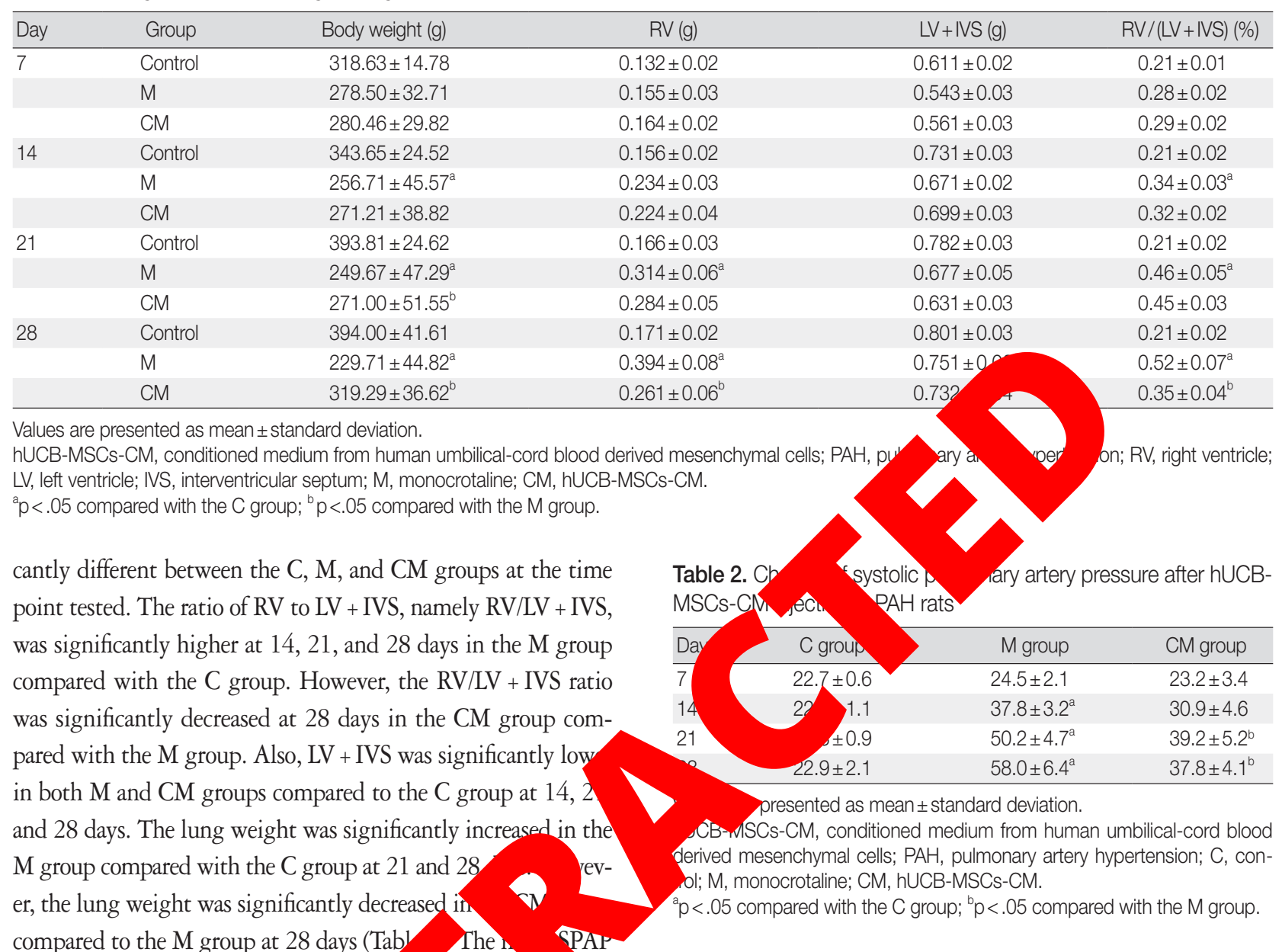

compared to the $\mathrm{M}$ group at 28 days (Tabl The SPAP was also significantly increased in the comparea the $\mathrm{C}$ and $\mathrm{CM}$ groups at 14,21 , and $?$ Cytokine profile in the lung tis treatment

A profile of the to investigate potent. nes he lung romogenates was made ryeon hUCB-MSC-CM treatment (Fig. 1). Ten pro-infic tory cytokines that included cytokine-induced neutrophil chemoattractant-1 (CINC-1), cytokine-induced neutrophil chemoattractant-2a/b (CINC-2a/b), chemokine (C-X-C motif) ligand 1 (CX3CL1), lipopolysaccharide-induced CXC chemokine (LIX), leukocyte endothelial cell adhesion molecule 1 (LECAM-1), chemokine (C-X-C motif) ligand 7, TIMP-1, vascular endothelial growth factor (VEGF), IL-1 $\alpha$, and CCL5 were examined in the C, M, and CM groups. CINC-1, CINC-2a/b, CX3CL1, LIX, LECAM-1, TIMP-1, and VEGF were lower in the M and CM groups, whereas TIMP-1, IL-1 $\alpha$, and CCL5 were higher in the CM group compared to the $\mathrm{C}$ and $\mathrm{M}$ groups. CCL7 was higher in the $\mathrm{M}$ group, whereas CCL7 was lower in the $\mathrm{CM}$ group compared to the $\mathrm{M}$ group (Fig. 1).

\section{Immunohistochemistry analysis of lung samples}

Immunohistochemistry (IHC) staining of the lung tissue revealed that TIMP-1-, IL-1 $\alpha$-, and CCL5-positive cells were more prevalent in the $\mathrm{CM}$ group, and then followed by the $\mathrm{M}$ group in comparison with the $\mathrm{C}$ group at 28 days (Fig. 2A-R). These results confirmed that hUCB-MSC-CM increased the expression of certain immunomodulating cytokines (at the protein level) in the lungs of treated animals. Three weeks after hUCB-MSCCM transfusion, TIMP-1-, IL-1 $\alpha$-, and CCL5-positive cells were still observed at the transplanted lung area in the CM group. The increased levels of TIMP-1, IL-1 $\alpha$, and CCL5 immunoreactivity observed in the $\mathrm{M}$ group were statistically significant $(\mathrm{p}<.05)$. The increased levels of CCL5 immunoreactivity were also significant in the CM group compared with the $\mathrm{M}$ group (Fig. 2S).

\section{Western blot analysis}

The protein expressions of CCL5 at 28 days were significant- 
ly increased in the $\mathrm{M}$ group compared to the $\mathrm{C}$ group. The protein expressions of TIMP-1, IL- $1 \alpha$, and CCL5 at 28 days were significantly increased in the $\mathrm{CM}$ group compared to the $\mathrm{M}$ group (Fig. 3). The protein expressions of caspase-3 and $\mathrm{Bcl}-2$ were significantly increased in the $\mathrm{M}$ group compared to the $\mathrm{C}$ group at 28 days. The protein expressions of caspase- 3 and $\mathrm{Bcl}-$
2 were significantly decreased in the CM group compared to the $\mathrm{M}$ group at 28 days (Fig. 4).

\section{TUNEL apoptosis assay}

The TUNEL staining was performed to detect apoptotic DNA in the lung tissue. The assayed $\mathrm{C}$ group did not have any positive
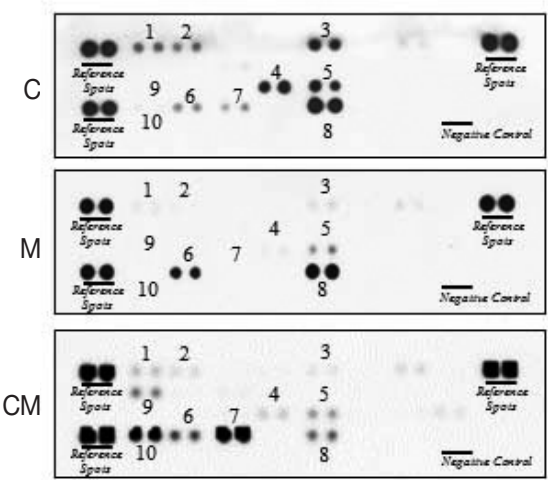

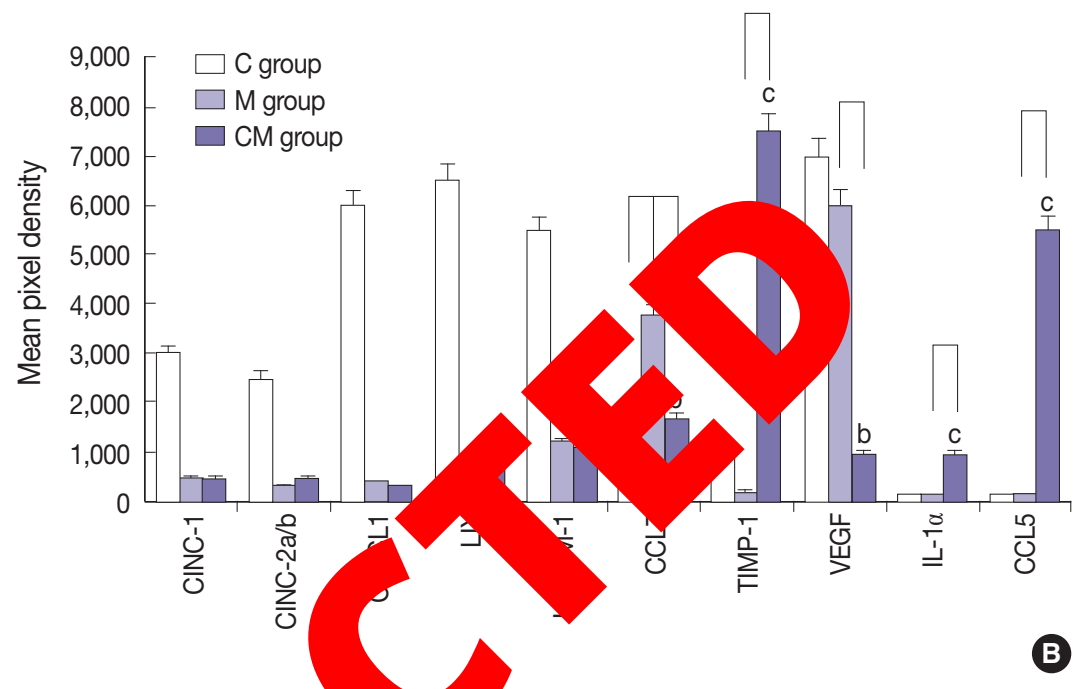

Fig. 1. Inflammatory cytokine expressions in the three groups. (A) T areen WI -MSCs-CM affect local production of inflammatory cytokines by lung cells in the three groups, a cytokine array is $\quad$ lung momogenates. (B) TIMP-1, IL-1 $\alpha$, and CCL5 are higher in the $\mathrm{CM}$ group compared to the $\mathrm{C}$ and $\mathrm{M}$ groups, whereas CCL $V$ ber in the CM group compared to the M group. CINC-1, CINC-2a/b, CX3CL1 LIX, and LECAM-1 are higher in the $\mathrm{C}$ group eans the $\mathrm{M}$ and CM groups. $\mathrm{C}$ group, control group $(\mathrm{n}=7) ; \mathrm{M}$ group, monocrotaline group ( $n=7)$; CM group, hUCB CM gn $n=7$ ). hUCB-MSCs-CM, conditioned medium from human umbilical-cord blood derived mesenchymal cells; CINC tok nduced utrophil chemoattractant-1; CINC-2a/b, cytokine-induced neutrophil chemoattractant-2a/b; CX3CL1, chemokin (C LIX, lipopolysaccharide-induced CXC chemokine; LECAM-1, leukocyte endothelial cell adhesion molecule 1; , che (C-U motif) ligand 7; TIMP-1, tissue inhibitor of metalloproteinase 1; VEGF, vascular endothelial growth factor; IL-1 $\alpha$ kin $1 \alpha$. ${ }^{2} \mathrm{p}$ sompared with the $\mathrm{C}$ group; ${ }^{b, c} \mathrm{p}<.05$ compared with the $\mathrm{M}$ group.

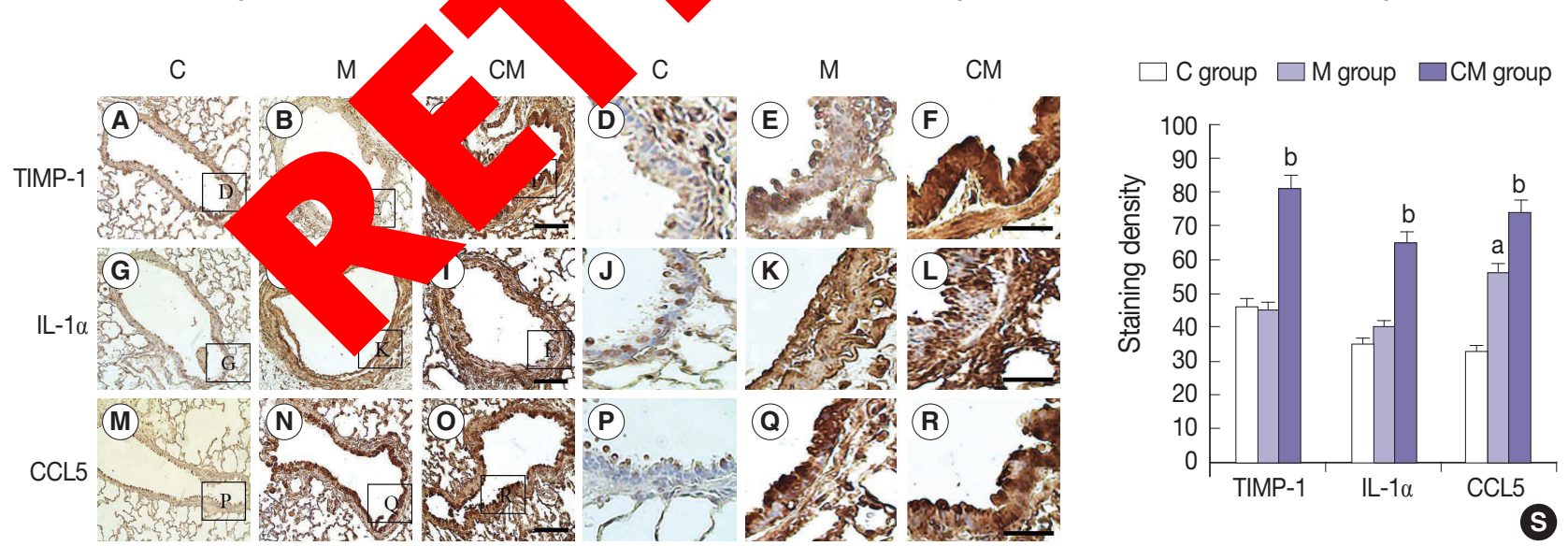

Fig. 2. Localization of IL-1 $\alpha$, CCL5, and TIMP-1-immunoreactive cells in the lung tissues at 28 days. (A-R) Immunohistochemical expression reveals that the positive cells of IL-1 $\alpha, C C L 5$, and TIMP-1 are significantly higher in the CM group than that in the $\mathrm{C}$ and M groups, and they are higher in the M group than that in the $\mathrm{C}$ group. (S) The increased levels of $\mathrm{LL}-1 \alpha$, CCL5, and TIMP-1 immunoreactivity observed in the CM group are statistically significant. The levels of IL-1 $\alpha$, CCL5, and TIMP-1 immunoreactivity are significantly decreased in the CM group compared with the $\mathrm{C}$ and $\mathrm{M}$ groups. Panels $\mathrm{A}-\mathrm{C}, \mathrm{G}-\mathrm{I}$, and $\mathrm{M}-\mathrm{O}$ are high power views of panels $\mathrm{D}-\mathrm{F}, \mathrm{J}-\mathrm{L}$, and $\mathrm{P}-\mathrm{R}$, respectively. C, control; M, monocrotaline; CM, hUCB-MSCs-CM; hUCB-MSCs-CM, conditioned medium from human umbilical-cord blood derived mesenchymal cells; TIMP-1, tissue inhibitor of metalloproteinase 1; IL-1 $\alpha$, interleukin $1 \alpha$; CCL5, chemokine (C-C motif) ligand $5 .{ }^{2} p<.05$ compared with the C group; ${ }^{b} p<.05$ compared with the M group. 

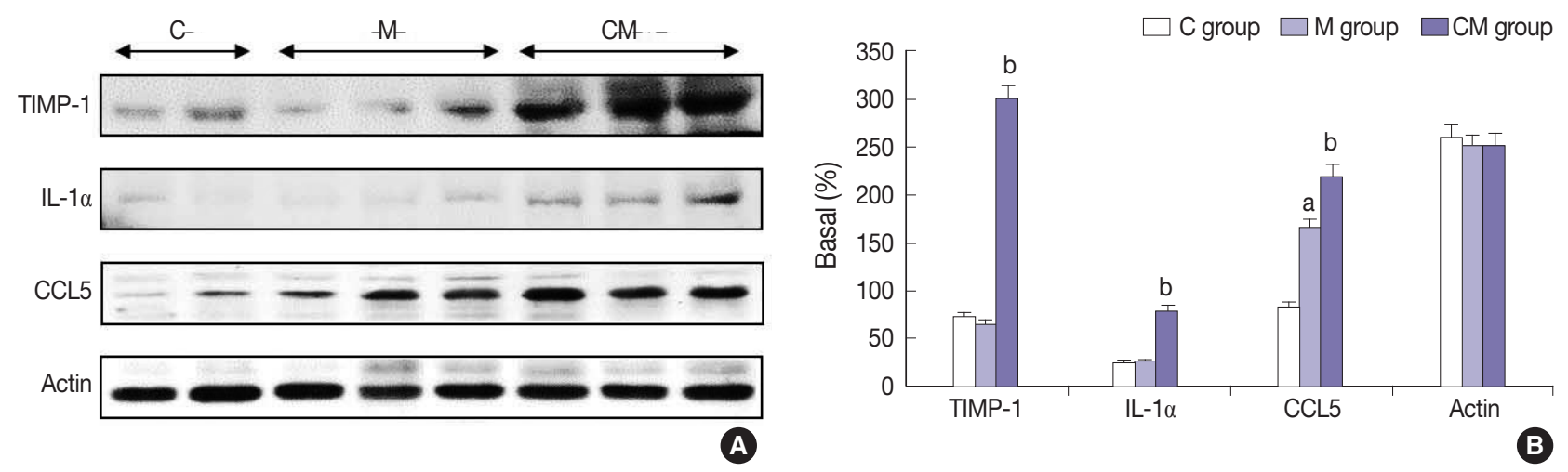

Fig. 3. Changes of IL-1 $\alpha, C C L 5$, and TIMP-1 protein expression levels after hUCB-MSCs-CM injection in P' These are pictures of protein expression levels of IL-1 $\alpha$, CCL5, and TIMP-1 in the lung tissues. (B) The protein expressions le of IL- $\quad$ CL5, and TIMP-1 at 28 days are significantly increased in the $\mathrm{CM}$ group compared to the $\mathrm{C}$ and $\mathrm{M}$ groups. The protein expro $\mathrm{C}$, re increased in the M group compared to the $\mathrm{C}$ group. C, control; M, monocrotaline; CM, hUCB-MSCs-CM; hUCB ds-Cly dit' medium from human umbilical-cord blood derived mesenchymal cells; TIMP-1, tissue inhibitor of metalloprotein
(C-C motif) ligand $5 .{ }^{a} \mathrm{p}<.05$ compared with the C group; ${ }^{b} \mathrm{p}<.05$ compared with the M grone.
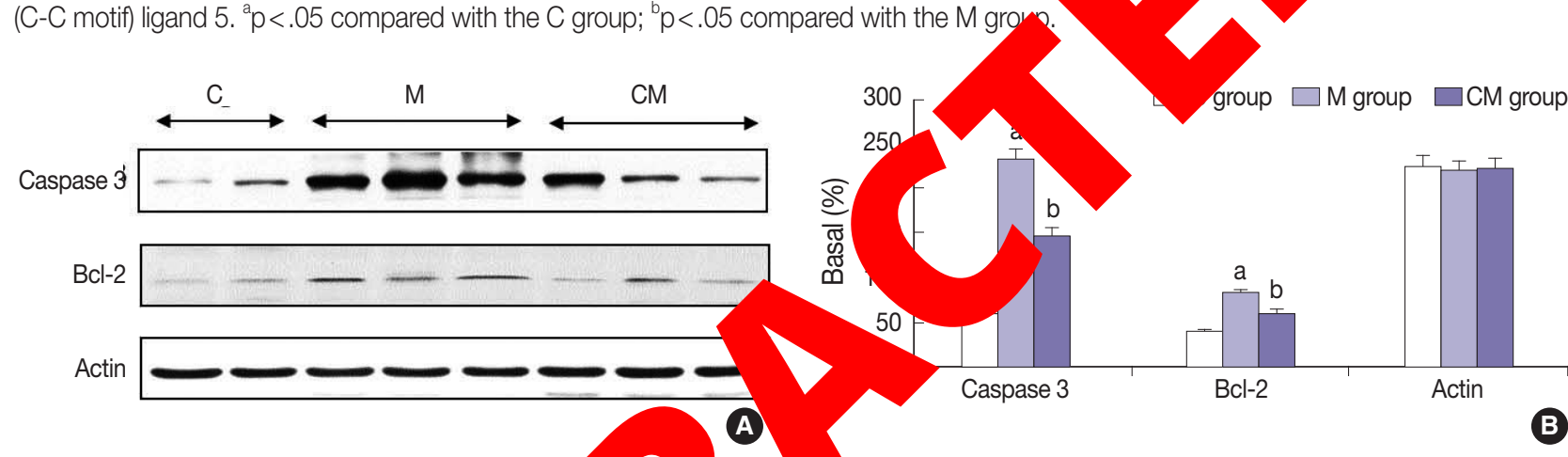

Fig. 4. Changes of caspase-3 and Bcl-2 protein 6 , Cor a tein expression levels of caspase-3 and $\mathrm{Bcl}-2$ the protein expressions levels of caspase-3 and $\mathrm{Bcl}-2$ at 28 days are significantly increased in the M group com to the ups. However, the protein expressions levels of caspase-3 and Bcl-2 are decreased in the CM group compared to up. C, co ol; M, monocrotaline; CM, hUCB-MSCs-CM; hUCB-MSCs-CM, conditioned medium from human umbilical-cord ${ }^{W}$ ood den sesenchymal cells. ${ }^{a} \mathrm{p}<.05$ compared with the $\mathrm{C}$ group; ${ }^{b} \mathrm{p}<.05$ compared with the $\mathrm{M}$ group.

staining (Fig. 5A, D). However, grod lung tissues with a positive TUN $\mathrm{g}$ as sc the presence of dark brown nuclei (Fig. $>$ also contained cells with brown nuclei, indicatin ptotic DNA (Fig. 5C, F). Apoptotic cells were significantly prevalent in the $\mathrm{M}$ group than in the $\mathrm{C}$ group, but they were less prevalent in the $\mathrm{CM}$ group than in the $\mathrm{M}$ group (Fig. 5G). The results indicated that hUCB-MSC$\mathrm{CM}$ could attenuate apoptosis in the lung tissues of treated $\mathrm{PAH}$ rats.

\section{DISCUSSION}

In this study, we tested the effects of CM infusion on PAH affected lung tissue in a rat model. It was previously demonstrated that CM of hUCB-MSCs contain active levels of a number of disease modifying growth factors and cytokines. ${ }^{21,22} \mathrm{CM}$ of
hUCB-MSCs contain sizable levels of angiopoietin, hepatocyte growth factor, interleukin-4, insulin-like growth factor, placental growth factor, vascular endothelial cell growth factor, angiogenin, stem cell factor, and tyrosine hydroxylase. ${ }^{5,23-25}$ Our previous studies demonstrated the neuroprotective effects of conditioned media from hADSC and hNSC in rat models of stroke and Huntington's disease. ${ }^{14,15}$ Therefore, we chose to test the CM prepared from hUCB-MSCs in a PAH rat model for therapeutic signals.

MSCs are multipotent stromal cells that have self-renewal capacity, and can differentiate into a variety of cell types such as osteoblasts, chondrocytes, myocytes, and adipocytes. ${ }^{26,27}$ MSCs have been isolated from several different sources such as embryonic tissue, bone marrow, adipose tissue, and the placenta. ${ }^{28}$ MSCs are the source of many immune-dampening cytokines and in this regard, have demonstrated potency in a number of disease mod- 
C

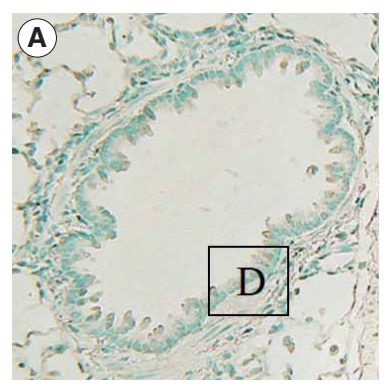

(D)

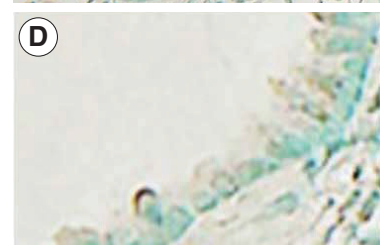

M

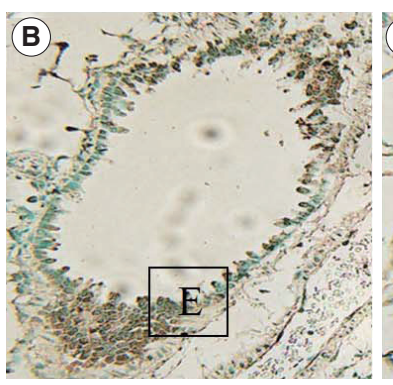

(E)

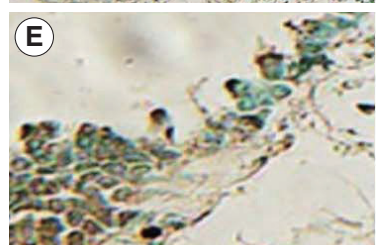

$\mathrm{CM}$

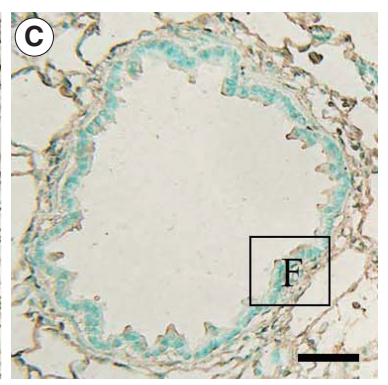

(F)

Fig. 5. TUNEL assay of lung tissues at 28 days after hUCB-MSCs-CM transfusion. (A-F) Imr positive cells of apoptosis are significantly higher in the M group than that in the C group; in the M group. (G) The increased levels of TUNEL immunoreactivity observed in the $M$ immunoreactivity are significantly decreased in the CM group compared with the $\mathrm{M}$ attenuate the vascular remodeling. Panels $\mathrm{A}-\mathrm{C}$ are high power views of panels $\mathrm{D}$ MSCs-CM; hUCB-MSCs-CM, conditioned medium from human umbilical-ce the $\mathrm{C}$ group; ${ }^{\mathrm{b}} \mathrm{p}<.05$ compared with the M group.

els. ${ }^{29,30}$ In addition the secreted factors from MSCs display antiapoptotic, proliferative activity and the cells may be involved the removal of harmful factors from their vicinity. ${ }^{23,31}$

From our study, we detected relatively high concep ions of CCL5, TIMP-1, and IL-1 $\alpha$ in hUCB-MSC-CM sues compared with MCT alone (M) and co confirmed by a rat cytokine array panel $(\mathrm{P}$ important roles in a number of biolog includ, $\delta$ innate immunity, apoptosis, angiog is, cell gh and differentiation. $^{32}$ These processes $\mathrm{p}$ tection and recovery.

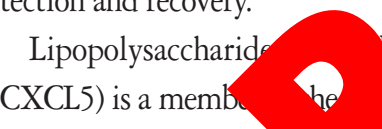
tent neutrophil chemoa curring inhibitor of metal ceinases, ${ }^{34-36}$ and TIMPs inhibit tumorigenesis, cellular invasion, metastasis, and angiogenesis. TIMPs may also promote tumor growth and inhibit apoptosis. These opposite roles of TIMPs in tumor regression and progression have been attributed to modulation by the tissue microenvironment. ${ }^{37}$ Many cytokines induce endothelial cells to express adhesion molecules and lead to secretion of chemokines that attract white blood cells to a site of injury. ${ }^{38,39}$ In our study, for the hUCB-MSC-CM treated PAH induced animals, the lung tissues showed significant increases in the number of IL- $1 \alpha$ positive pulmonary arterioles compared with the control group. IL- $1 \alpha$ (and also tumor necrosis factor $\alpha$ ) are known to stimu-

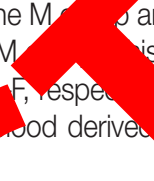

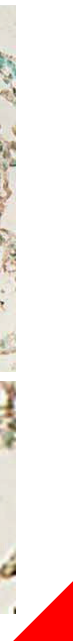

. . are sta is result in ates that hUCB-MSCS-CM could C, control; M, monocrotaline; CM, hUCBenchymal cells. ${ }^{a} \mathrm{p}<.05$ compared with $\square$ C group $\square$ M group $\square$ CM group

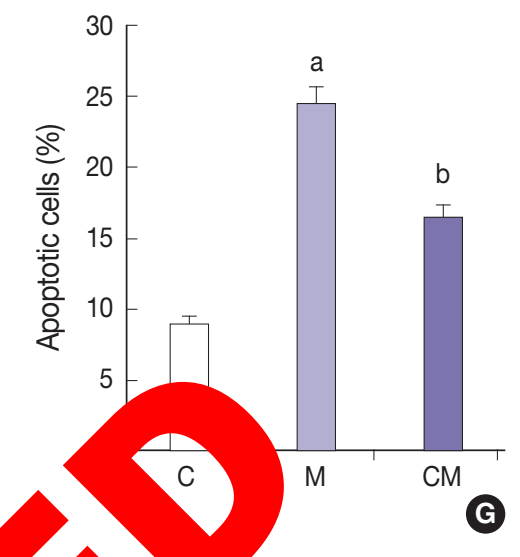


treatment for PAH and bypass several technical limitations of a direct MSC cell transplantation. The present study also revealed certain changes in chemokine, cytokine, and growth factor levels after hUCB-MSC-CM transfusion in a PAH rat model. Through a complex interaction of these mediators involved in immunomodulation and inflammation, we may expect a positive effect on reducing the impact of PAH on lung cells. Exactly how these cytokines and factors interact to impact the survival of the lung tissue cells remains to be explored. As there are several treatment options available for $\mathrm{PAH}$ in people, an effective therapy in prolonging survival remains elusive. Our data with factors present in hUCB-MSC-CM may present an exciting opportunity for more effective therapies.

The limitations of our study included the small sample size and a short follow-up of the treated animals. Future studies with larger sample sizes and a longer duration of treatment will be required, along with standardizing the quality and amount of hUCB-MSC-CM, frequency, and the duration required for the treatment.
7. Zhao YD, Courtman DW, Ng DS, et al. Microvascular regeneration in established pulmonary hypertension by angiogenic gene transfer. Am J Respir Cell Mol Biol 2006; 35: 182-9.

8. Saigawa T, Kato K, Ozawa T, et al. Clinical application of bone marrow implantation in patients with arteriosclerosis obliterans, and the association between efficacy and the number of implanted bone marrow cells. Circ J 2004; 68: 1189-93.

9. Kang H, Kim KH, Lim J, et al. The therapeutic effects of human mesenchymal stem cells primed with sphingosine-1 phosphate on pulmonary artery hypertension. Stem Cells Dev 2015; 24: 1658-71.

10. Umar S, de Visser YP, Steendijk P llogenic stem cell therapy improves right ventricular fy ving lung pathology in rats with pulmonary hyp vion. A Physiol Heart Circ Physiol 2009; 297: H

11. Kim AK, Kim MH, Stem- herapy for peripheral arterial occlus sease. $\quad$ Va dovasc Surg 2011; 42: 667-75.

12. Lee $\mathrm{KB}$ Kim $\mathrm{AK}$, stem cell therapy in patients with thro boangiit rans: assessment of the long-term clinical out1e and analysis e prognostic factors. Int J Stem Cells 2011; 4: 98.

13.

\section{Conflicts of Interest}

No potential conflict of interest relevant to this article reported.

\section{Acknowledgments}

This work was supported by a Korea Uniy

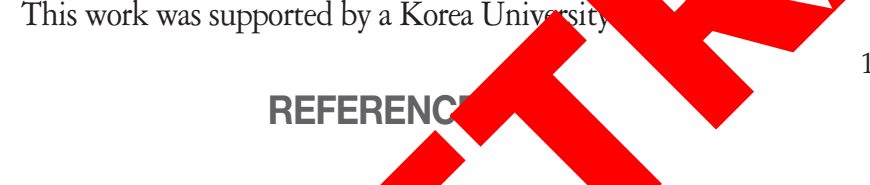

1. Farber HW, Loscalzo J. Pulm art l hyperte on. N Engl J Med 2004; 351: 1655-65.

2. Liang OD, Mitsialis $\mathrm{g} \mathrm{Ms}$, esenchymal stromal cells expressing axy a 1 reverse pulmonary hypertension. Stem Cells 2011,

3. Can MM, Tanboga IH, D n HC, et al. Enhanced hemostatic indices in patients with pulmonary arterial hypertension: an observational study. Thromb Res 2010; 126: 280-2.

4. Fukumoto $Y$, Shimokawa H. Recent progress in the management of pulmonary hypertension. Circ J 2011; 75: 1801-10.

5. Liu Q, Luo Z, He S, et al. Conditioned serum-free medium from umbilical cord mesenchymal stem cells has anti-photoaging properties. Biotechnol Lett 2013; 35: 1707-14.

6. Lee JC, Kim KC, Yang YS, et al. Microarray analysis after umbilical cord blood derived mesenchymal stem cells injection in monocrotaline-induced pulmonary artery hypertension rats. Anat Cell Biol 2014; 47: 217-26. NR, Park KB, et al. A phase I study of human cord
blooa-umed mesenchymal stem cell therapy in patients with peal arterial occlusive disease. Int J Stem Cells 2013; 6: 37-44.

4. Cho YJ, Song HS, Bhang S, et al. Therapeutic effects of human adipose stem cell-conditioned medium on stroke. J Neurosci Res 2012; 90: 1794-802.

15. Lim HC, Lee ST, Chu K, et al. Neuroprotective effect of neural stem cell-conditioned media in in vitro model of Huntington's disease. Neurosci Lett 2008; 435: 175-80.

16. Lee EJ, Park SJ, Kang SK, et al. Spherical bullet formation via Ecadherin promotes therapeutic potency of mesenchymal stem cells derived from human umbilical cord blood for myocardial infarction. Mol Ther 2012; 20: 1424-33.

17. Pereira T, Ivanova G, Caseiro AR, et al. MSCs conditioned media and umbilical cord blood plasma metabolomics and composition. PLoS One 2014; 9: e113769.

18. Lipke DW, Arcot SS, Gillespie MN, Olson JW. Temporal alterations in specific basement membrane components in lungs from monocrotaline-treated rats. Am J Respir Cell Mol Biol 1993; 9: 418-28.

19. Bieback K, Kern S, Kluter H, Eichler H. Critical parameters for the isolation of mesenchymal stem cells from umbilical cord blood. Stem Cells 2004; 22: 625-34.

20. Wang M, Yang Y, Yang D, et al. The immunomodulatory activity of human umbilical cord blood-derived mesenchymal stem cells in vitro. Immunology 2009; 126: 220-32.

21. Jeong SY, Kim DH, Ha J, et al. Thrombospondin-2 secreted by hu- 
man umbilical cord blood-derived mesenchymal stem cells promotes chondrogenic differentiation. Stem Cells 2013; 31: 2136-48.

22. Kim JY, Kim DH, Kim JH, et al. Soluble intracellular adhesion molecule-1 secreted by human umbilical cord blood-derived mesenchymal stem cell reduces amyloid-beta plaques. Cell Death Differ 2012; 19: 680-91.

23. Jin H, Sanberg PR, Henning RJ. Human umbilical cord blood mononuclear cell-conditioned media inhibits hypoxic-induced apoptosis in human coronary artery endothelial cells and cardiac myocytes by activation of the survival protein Akt. Cell Transplant 2013; 22: 1637-50.

24. Yang S, Sun HM, Yan JH, et al. Conditioned medium from human amniotic epithelial cells may induce the differentiation of human umbilical cord blood mesenchymal stem cells into dopaminergic neuron-like cells. J Neurosci Res 2013; 91: 978-86.

25. Yang C, Lei D, Ouyang W, et al. Conditioned media from human adipose tissue-derived mesenchymal stem cells and umbilical cord-derived mesenchymal stem cells efficiently induced the apoptosis and differentiation in human glioma cell lines in vitro. Biomed Res Int 2014; 2014: 109389.

26. Jiang Y, Jahagirdar BN, Reinhardt RL, et al. Pluripotency of mesenchymal stem cells derived from adult marrow. Nature 2002; 41-9.

27. Mareschi K, Ferrero I, Rustichelli D, et al. Expansion mal stem cells isolated from pediatric and adult row. J Cell Biochem 2006; 97: 744-54.

28. Secco M, Zucconi E, Vieira NM, et al. M umbilical cord: cord is richer than bloo

29. Kim ES, Jeon HB, Lim H, et al. umbilical cord blood-derive lanogenesis by promoting protec. One 2015; 10: e0128

30. Kim JY, Kim DH DS bilical cord blood-den tesentrymal stem cells reduces amyloid-beta42 neurotoxicity h d. FEBS Lett 2010; 584: 3601-8.

31. Flynn A, Barry F, O’Brien T. UC blood-derived mesenchymal stromal cells: an overview. Cytotherapy 2007; 9: 717-26.
32. Dranoff G. Cytokines in cancer pathogenesis and cancer therapy. Nat Rev Cancer 2004; 4: 11-22

33. Chandrasekar B, Melby PC, Sarau HM, et al. Chemokine-cytokine cross-talk. The ELR+ CXC chemokine LIX (CXCL5) amplifies a proinflammatory cytokine response via a phosphatidylinositol 3-kinase-NF-kappa B pathway. J Biol Chem 2003; 278: 4675-86.

34. Brew K, Dinakarpandian D, Nagase H. Tissue inhibitors of metalloproteinases: evolution, structure and function. Biochim Biophys Acta 2000; 1477: 267-83.

35. Bourboulia D, Stetler-Stevenson WG. Matrix metalloproteinases (MMPs) and tissue inhibitors of moroteinases (TIMPs): Positive and negative regulators Biol 2010; 20: 161-8.

36. Jiang Y, Goldberg ID $\mathrm{IE}$. CO, of tissue inhibitors of metalloproteinases ncogen,.002; 21: 2245-52.

37. Stetler-Stey WG. itors of metalloproteinases in cell sign alloprote - Independent biological activities. Scionnar 2008

38 G. MB, Randor. Chemokines and tissue injury. Am J hol 1995; 1M. 6: 1287-301.

39. Leger AJ, Kuliopulos A. Therapeutic targeting of molecumo involved in leukocyte-endothelial cell interactions. FEBS 273: $4416-24$

. Kolb M, Margetts PJ, Anthony DC, Pitossi F, Gauldie J. Transient expression of IL-1beta induces acute lung injury and chronic repair leading to pulmonary fibrosis. J Clin Invest 2001; 107: 1529-36.

41. Johnston CJ, Piedboeuf B, Rubin P, Williams JP, Baggs R, Finkelstein JN. Early and persistent alterations in the expression of interleukin-1 alpha, interleukin-1 beta and tumor necrosis factor alpha mRNA levels in fibrosis-resistant and sensitive mice after thoracic irradiation. Radiat Res 1996; 145: 762-7.

42. Phan SH, Kunkel SL. Lung cytokine production in bleomycin-induced pulmonary fibrosis. Exp Lung Res 1992; 18: 29-43.

43. Pan LH, Ohtani H, Yamauchi K, Nagura H. Co-expression of TNF alpha and IL-1 beta in human acute pulmonary fibrotic diseases: an immunohistochemical analysis. Pathol Int 1996; 46: 91-9. 